\title{
SOLAR ACTIVITY AND REGIONAL CLIMATE
}

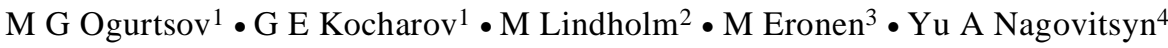

ABSTRACT. We performed a statistical analysis of the data on summer temperature anomalies in northern Fennoscandia (81995 AD) and found that a 70-130-yr cycle is present in this series during most of the time period. A comparison of the reconstructed northern Fennoscandia temperature with different indicators of solar activity (Wolf numbers, the length of solar Schwabe cycle, extended bi-decadal radiocarbon series, and data on sunspots observed by naked eye) shows that the more probable cause of the periodicity is the modulation of regional northern Fennoscandia climate by the long-term solar cycle of Gleissberg. The effect of this century-scale solar modulation of the global Northern Hemisphere temperature is weaker.

\section{INTRODUCTION}

The question of the existence of a solar-climate link and its possible physical mechanism is of interest in geophysics. Although meteorologists often doubt the possibility of the Sun affecting the lower atmosphere (Monin 1969; Pittock 1978) many recent results confirm the reality of solar-climate link. E.g., Mitchell et al. (1979) have shown the presence of 22-yr solar cycle in the rhythm of droughts in the eastern USA after 1610 AD. Veretenenko and Pudovkin (1993) and Pudovkin and Veretenenko (1996) have shown the connection between the variation of the cosmic-ray intensity and the frequency of cirrus clouds occurrence. Also, Beer et al. (1994) found a good phase coherency between secular variations in the Northern Hemisphere temperature and concentration of cosmogenic ${ }^{10} \mathrm{Be}$ in Greenland ice (the quantity reflects the activity of the sun). An interesting idea was suggested by FriisChristensen and Lassen (1991) — they found a negative correlation between the global land temperature in the Northern Hemisphere and the length of solar Schwabe cycle. The same relationship with the solar cycle length was reported for the regional northern Finland temperature by Ulikh (1996).

It should be noted that the finding of Friis-Christensen and Lassen (1991) needs further study, because it was subjected to serious criticism by Damon and Peristykh (1999). The ideas of Svensmark and Friis-Christensen (1997), which concerned the correlation between the cosmic-ray intensity and global cloudiness, are now actively being debated (Jorgensen and Hansen 2000; Gierens and Ponater 1999; Swensmark and Friis-Christensen 1999). The possible influence of changes in solar irradiation and its spectral content is also the subject of intensive investigation (Hoyt and Schatten 1997; Beer et al. 2000). Although evidence for a solar-climate connection has been found already, the mechanism of solar influence on the Earth's atmosphere has not been clarified yet, despite some other solutions proposed for this problem (Shindell et al. 1999; Tinsley and Heelis 1993).

Therefore, further investigation of possible solar action on climate, its character and evolution at different time scales is of great importance. Recently, the annual reconstruction of anomalies in summer temperature in northern Fennoscandia (NFST) at 8-1995 AD was accomplished at the University of Joensuu (Finland). This series, shown in Figure 1A, was obtained using tree-ring data. It provides us with a unique opportunity to trace the temperature variations on a long time scale (about 2000 years). In this work, this aim was achieved by a thorough statistical analyses of the NFST data set using different statistical methods.

\footnotetext{
${ }^{1}$ A.F. Ioffe Physico-Technical Institute of the Russian Academy of Sciences, 194021, Polytechnicheskaya 26, St.-Petersburg, Russia. Email: maxim.ogurtsov@pop.ioffe.rssi.ru.

${ }^{2}$ Saima Center for Environmental Sciences, Linnankatu 11, FIN-57130 Savonlinna, Finland

${ }^{3}$ University of Helsinki, Department of Geology, POB 11, FIN-00014, Helsinki, Finland

${ }^{4}$ Central Astronomy Observatory, Pulkovo,196140, St.-Petersburg, M-140, Russia
}

(C) 2001 by the Arizona Board of Regents on behalf of the University of Arizona

Radiocarbon, Vol 43, Nr 2A, 2001, p 439-447

Proceedings of the 17th International ${ }^{14} \mathrm{C}$ Conference, edited by I Carmi and E Boaretto 


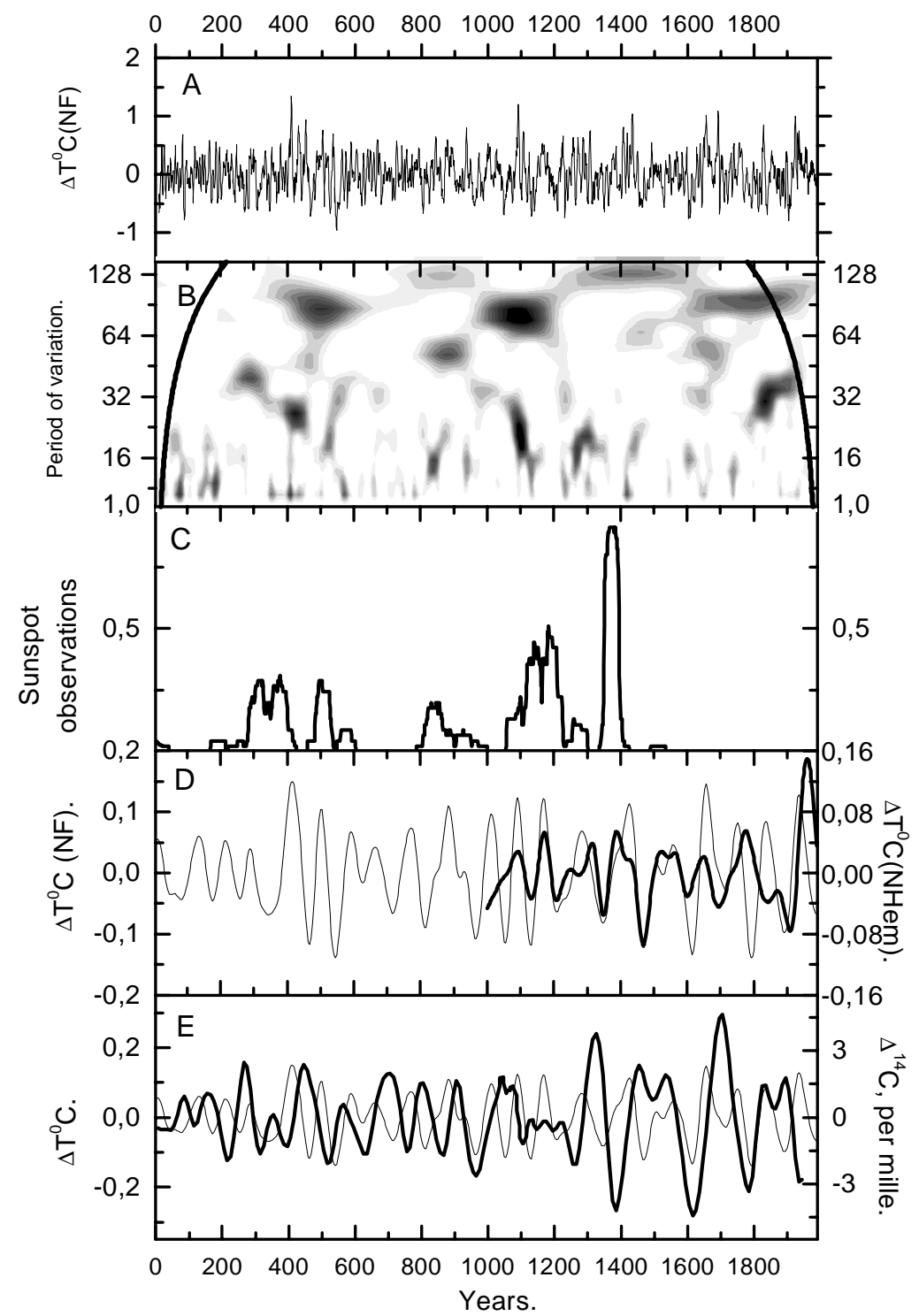

Figure 1 A-northern Fennoscandia summer temperature (5-yr moving averaged), B-normalized wavelet spectrum of NFST (white domains $=w^{2}(a, t)<1.0$, black domains $=\mathrm{w}^{2}(\mathrm{a}, \mathrm{t})>7.0, \mathrm{C}-$ number of sunspot observations made by naked eye (45-yr averaged), Dwavelet filtered ( $\mathrm{T}_{\mathrm{pass}}=76-128 \mathrm{yr}$ ) NFST (thin curve) and NHST (thick curve), E-wavelet filtered $\left(\mathrm{T}_{\text {pass }}=76-128 \mathrm{yr}\right.$ ) NFST (thin curve) and $\Delta^{14} \mathrm{C}$ (thick curve). $\Delta{ }^{14} \mathrm{C}$ is shifted by $30 \mathrm{yr}$.

\section{METHODS}

\section{Methods of Data Acquisition}

It is well known that the width of tree rings at upper and northern tree lines depends mainly on the temperature of the growth period. This relationship allows the use of the tree-ring data as a summer temperature proxy. In Lindholm and Eronen (2000), the July temperature in northern Fennoscandia 
was reconstructed for 8-1995 AD by using a master ring-width chronology of Scots pine (Pynus silvestris L.). The chronology was constructed from 93 living trees and 275 dead trees collected between $68^{\circ} \mathrm{N}$ and $70^{\circ} \mathrm{N}$ (northern tree limit), and between $20^{\circ} \mathrm{E}$ and $30^{\circ} \mathrm{E}$. Standardization of different chronologies was made by using negative exponential functions and, alternatively, regression lines. It should be noted that the procedure of standardization could lead to a suppression of low-frequency variability.

The reconstructed temperature was compared with the real temperature data at 1911-1988 AD and it was found that the tree-ring reconstruction explains $0.4-0.57$ of real temperature variance. This result shows the reliability of the reconstruction made by Lindholm and Eronen (2000).

\section{Methods of the Statistical Processing of the Data}

Our first task was to analyze the spectral content of NFST and its evolution in time. To extract the information from the NFST data set, analyses using both Fourier and wavelet approaches were performed. The main shortcoming of Fourier methods, applied in time-series analyses for a long time, is their assumption of the stationarity of process. In case of non-stationary data set (the class of data sets which the main part of climatic series belongs to) the Fourier transform can evaluate only the average power at a given frequency and cannot trace the variation of spectral content in time. This lack is absent when a continuous wavelet transform is used. Wavelet transform has a principal difference with Fourier transform - the analyzed signal here is decomposed not to infinite sinusoidal harmonics but to a number of orthogonal waves of solyton type. These waves are called wavelets and they are well localized both in frequency and in time, while Fourier harmonics are localized only in frequency. Thanks to this property, the wavelet transformation is suitable for analyses of non-stationary time series, including non-periodic inhomogenieties, deterministic chaos, and local periodic structures (Astafieva 1996). The wavelet method, intensively developed lately (Grossman and Morlett 1984; Mallatt 1989; Percival 1995; Astafieva 1996; Torrence and Compo 1998) is based on the investigation of $w(a, t)$ - the coefficients of wavelet transform of the signal under investigation

$$
w(a, t)=a^{-1 / 2} \int_{-\infty}^{+\infty} f\left(t^{\prime}\right) \psi^{*}\left(\frac{t-t^{\prime}}{a}\right) d t
$$

where $f\left(t^{\prime}\right)=$ the analyzed signal, $\psi(t)=$ the analyzing wavelet, $a=$ the scale parameter of analyzing wavelet, determines its dilation, and $t^{\prime}$ determines the translation of $\psi(t)$.

In our work we used the real MHAT (Mexican hat)-basis, which has a form of

$$
\psi_{\text {MHAT }}=\exp \left(-x^{2} / 2\right) *\left(1-x^{2}\right)
$$

and the complex basis of Morle

$$
\psi_{\text {Morle }}(x)=\pi^{-1 / 4} * \exp \left(-x^{2} / 2\right) * \exp \left(-i k_{0} x\right)
$$

where $\mathrm{k}_{0}$ is equal to 6.0 .

The set of scales $a_{\mathrm{j}}$ was chosen according to Torrence and Compo (1998)

$$
\begin{gathered}
a_{j}=a_{0} * 2^{\mathrm{j} \delta \mathrm{j}}-\text { the time scale, } j=0,1, \ldots, \mathrm{J} ; \\
\left.J=\delta j^{-1} * \log _{2}\left(N * \delta t / s_{0}\right)\right),
\end{gathered}
$$


where: $\delta t=$ the time step of series, $N=$ number points in series, $\delta j$ determines the time scale step, and $s_{0}$ determines the minimal time scale (usually $s_{0}=2 * \delta t$ ).

For a more convenient comparison of different wavelet spectra, the spectrum of each series was normalized by its variance. Another useful application of the wavelet technique is its filtration opportunity. The wavelet-filtered series is constructed according to formula of Torrence and Compo (1998)

$$
f(t)_{f i l t r}=\frac{\delta j * \delta t^{1 / 2}}{C_{\delta} * \psi(0)} * \sum_{j=j_{1}}^{j=j_{2}} \frac{\operatorname{Re}\left(w\left(a_{j}, t\right)\right)}{\sqrt{a_{j}}}
$$

where $C_{\delta}$ is the reconstruction factor.

In the present work we applied the wavelet filtration (MHAT basis was used, so $C_{\delta}=3.541$, $\psi(0)=0.867)$ for the investigation of phase relationship between different data sets on selected time scale.

\section{RESULTS}

Wavelet spectrum of NFST, obtained with using the basis of Morle is shown in the Figure 1B. It is seen that the long-term (70-130 yr) periodicity exists in the analyzed data set during few time intervals: 70-100 yr variation is observed during 380-600 AD and 1000-1200 AD, more weak 110130 -yr variation is present during $\sim 800-950 \mathrm{AD}$ and $~ 1350-1600 \mathrm{AD}, 90-110$-yr variation appears after the second part of 17th century. Fourier spectra of NFST calculated for the corresponding time intervals (410-700 AD, 1000-1500 AD, 1590-1990 AD) also contain peaks at periods of $85.1 \mathrm{yr}$ and $103.7 \mathrm{yr}$, respectively. The significance of these variations was estimated on the base of comparison with the red noise $(\alpha=0.3)$ continuum and confidence levels were found higher than 0.95 . Such periodicities are very similar to those of the solar activity. Actually, Damon and Peristykh (2000) found significant 88 -yr and 104-yr variation in bi-decadal ${ }^{14} \mathrm{C}$ data extending back over 11 millennia. The first period is more likely the manifestation of the solar cycle revealed by Gleissberg (1944) and another one can be attributed to the second harmonic of Suess cycle (Damon and Sonett 1991). So, the hypothesis about the solar origin of century-scale variations in NFST was checked. In order to analyze the possible connection between the centennial variation in NFST and the activity of the sun we compared this series with the direct indicators of solar activity:

1. Sunspot numbers (1700-1995 AD). The Wolf numbers, wavelet filtered in 76-128-yr band, are plotted in the Figure 2A.

2. The length of solar Schwabe cycle (SCL). The data on the length of individual cycles were taken from NGDC catalogue (directory ftp://ftp.ngdc.noaa/gov/SOLAR_DATA/ SUNSPOT_ NUMBERS/maxmin, see also Waldmeier [1961]). This value, smoothed by 5 points, is shown in the Figure 2B. As Figure 2B shows, the phase relationship between NFST and SCL is more reasonable than in the previous case.

Also we compared NFST with the variety of proxy data:

1. The extended $\Delta^{14} \mathrm{C}$ record derived from the bi-decadal tree-ring samples (Stuiver and Pearson 1993) and covers the time interval back to 7950 BP. It was established by Stuiver et al. (1991) that the main source of the century type radiocarbon variations is a solar modulation of cosmic ray flux. This series, wavelet filtered in 76-128-yr band, is shown in Figure 1E. 
2. Data on summer temperature in Northern Hemisphere (NHST) reconstructed by Mann et al. (1999) for 1000-1990 AD. Mann et al. (1999) show that the Fourier spectrum of the series has some power in secular frequency band. The set of data on Northern Hemisphere summer temperature, wavelet filtered in the 76-128-yr band, is shown in Figure 1D together with the NFST series, filtered by the same way.

3. Catalog of ancient oriental observations of sunspots made by naked eye (Wittman and $\mathrm{Xu}$ 1987). Nagovitsyn (2001) shows that the data on sunspots observed by naked eye (SONE) are quite a reliable indicator of long-term variations of solar activity in the past. Here we used the part of the catalog that covers the interval 0-1500 AD. These data, averaged over $45 \mathrm{yr}$, are shown in Figure 1C.

4. Finally, we compared data on historic minima and maxima of solar activity established by Schove (1983) using a variety of proxy data.

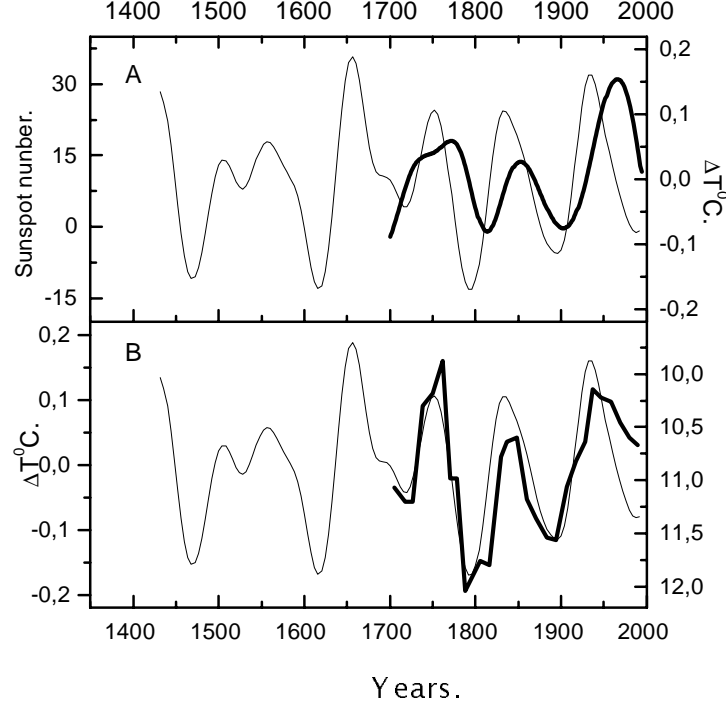

Figure 2 A-wavelet filtered $\left(\mathrm{T}_{\text {pass }}=76-128 \mathrm{yr}\right)$ NFST (thin curve) and Wolf numbers (thick curve), $\mathrm{B}$-wavelet filtered $\left(\mathrm{T}_{\text {pass }}=76-128 \mathrm{yr}\right)$ NFST (thin curve) and averaged length of solar Schwabe cycle).

The comparison of NFST series with the data sets described above led us to the following results:

1. The long-term (70-130 yr) variation of NFST after 1700 AD is in a good phase coherency with direct indicators of solar activity. But when we use the Wolf numbers, we see an effect similar to that found for a Northern Hemisphere land air temperature by Friis-Christensen and Lassen (1991) - the long-term cycle in NFST leads the same variation in solar activity with a time lag 15-25 yr (for the Northern Hemisphere land air temperature this value is about 20 years). When SCL is used, the phase relationship between temperature and solar activity is more reasonable.

2. The anticorrelation between filtered NFST and $\Delta^{14} \mathrm{C}$ is more moderate. But here we cannot anticipate an excellent anticorrelation, because among the century-type variations of radiocarbon concentration the 200 -yr cycles dominates (Stuiver et al. 1993). It is connected with the properties of carbon exchange system, which works as a low-pass filter and suppresses the variations with the shorter periods. The procedure of tree-ring data standardization, by contrast, leads to a suppression of the 200-yr cycle in reconstructed temperature. Moreover, the strong noise component, contained in both series weakens the correlation. But in any case, Figure $1 \mathrm{E}$ demonstrates that the long-term cycles in NFST and $\Delta^{14} \mathrm{C}$ anticorrelate satisfactorily at least during some part of full 2000-yr interval. Analyses show that the coefficient of negative correlation 
between century-long variations in NFST and ${ }^{14} \mathrm{C}$ concentration achieves 0.33 during $630-1530$ $\mathrm{AD}$ with a time lag $\sim 30 \mathrm{yr}$ (correlation is significant at $0.95 \mathrm{c}$.1.). This time lag likely is connected with the following phenomena. First, the long-term variation in summer surface temperature, both global and Fennoscandian, outstrips that in solar activity by $15-25$ yr. Second, $80-100-y r$ variation in ${ }^{14} \mathrm{C}$ concentration lags behind that in solar activity by 10-12 yr (Dergachev and Veksler 1991; Kocharov et al. 1981). The sum of these values gives us the 30 -yr shift.

3. The correlation between long-term variations in NFST and that in global Northern Hemisphere temperature also is ambiguous. Analyses show that the coefficient of correlation between these series, smoothed by $50 \mathrm{yr}$, attain the value about 0.4 with the lag $0-2 \mathrm{yr}$. Significance of the correlation was estimated using the statistical experiment based on the randomization of initial data sets. By means of this method, the long-term correlation between NFST and NHST was found to be significant at 0.97 c.l.

4. The long-term cycle in NFST has a tendency to increase during epochs of solar activity maxima that is illustrated in the Table 1. We used as the solar proxies the data on SONE (Wittman and Xu 1987) and the data of Schove (1983).

Table 1 Connection between the time evolution of the century-scale cycle in NFST and that in solar activity

\begin{tabular}{ccc}
\hline $\begin{array}{c}\text { Periods of long-term } \\
\text { NFST cycle (AD) }\end{array}$ & $\begin{array}{c}\text { Periods of maximal } \\
\text { solar activity }\end{array}$ & Periods of maximal solar activity \\
\hline$\cong 380-600$ & $\cong 450-600$ & $\cong 500-600$, Roman maximum \\
$\cong 800-950$ & $\cong 800-1000$ & \\
$\cong 980-1200$ & $\cong 1050-1270$ & $\cong 1100-1200$, Medieval maximum \\
$\cong 1350-1600$ & $\cong 1350-1380$ & $\cong 1350-1400$, late Medieval maximum \\
\hline
\end{tabular}

${ }^{\mathrm{a} A c c o r d i n g}$ to catalogue of Wittman and Xu (1987)

${ }^{b}$ According to Schove (1983)

The amplification of the long-term cycle during the modern maximum of solar activity continues the tendency described.

The next question is to clarify whether the effect revealed is local or global. To answer this question we analyzed the average summer temperature in Northern Hemisphere (NHST) reconstructed by Mann et al. (1999) for 1000-1990 AD (Figure 3A, above). As seen from the wavelet spectrum of NHST (Figure 3B), the secular variability is relevant for NHST also. Fourier analyses shows that at least during 1300-1800 AD, NHST has the significant time variation with a period close to $128 \mathrm{yr}$ (c.l. larger than 0.95). But comparison of long-term components of NHST and Wolf numbers (Figure 3D) does not demonstrate a persistent link between these values. As Figure 3D shows, the appreciable difference between two filtered data sets takes place in the middle of 19th century. Its possible cause may be connected with the influence of ocean-atmosphere processes related to the North Atlantic thermocycline circulation (Damon and Peristykh 1999). So, the solar induced 70-130-yr variation in summer temperature seems to be rather a local effect, takes place in Northern Fennoscandia and less manifested in a global Northern Hemisphere temperature. 


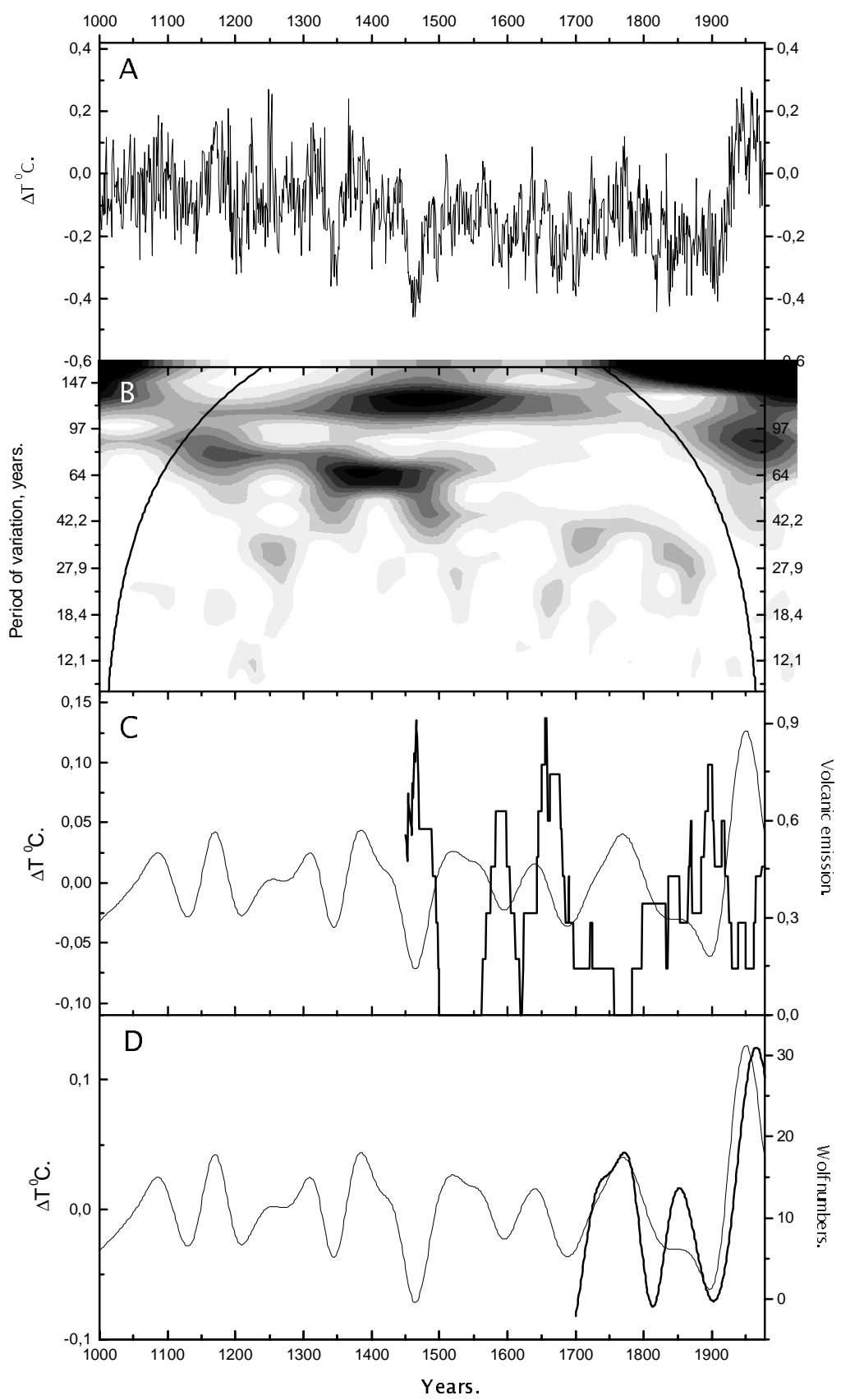

Figure 3 A-Northern Hemisphere Summer temperature (Mann et al. 1999), B-normalized wavelet spectrum of NFST (white domains $-\mathrm{w}^{2}(\mathrm{a}, \mathrm{t})<1.0$, black domains $-\mathrm{w}^{2}(\mathrm{a}, \mathrm{t})>10.0, \mathrm{C}-$ wavelet filtered ( $\mathrm{T}_{\text {pass }}=76-128 \mathrm{yr}$ ) NHST (thin curve) and Wolf numbers (thick curve). 


\section{CONCLUSIONS AND DISCUSSION}

Thorough statistical processing of the NFST data set and its comparison with a variety of direct and indirect indicators of solar activity led us to the following conclusions:

1. Solar forcing of regional climate in northern Fennoscandia during last $2000 \mathrm{yr}$ really exists. It manifests itself as a 70-130-yr cycle of solar origin, which appears periodically and is observed during the large part of full 2000-yr interval.

2. The connection between the century-scale variability in summer temperature and in solar activity takes place in northern Fennoscandia, and seems to be a regional effect. The long-term variations in global northern Hemisphere temperature more probably are controlled by many factors, not only by activity of the sun.

The mechanism of such solar-climate relationship needs to be clarified. The model, developed in Shindell et al. (1999), opens a new way to the solution of the problem. The model takes into consideration the dynamic coupling between the stratosphere and troposphere. In the model, the influence of solar irradiation on the stratospheric circulation via change in ozone chemistry is followed by the subsequent penetration of circulation changes into the troposphere. Finally, the solar forcing of the surface winds and sea level pressures is achieved. According to the model, the increase in solar irradiation leads to a corresponding increase in warm air inflow from southern latitudes to the north and respective zonal surface warming northward of $55^{\circ}$ and cooling from about $35^{\circ}$ to $55^{\circ}$ without any changes in mean global temperature. This effect can explain the solar induced climatic variations in the Arctic, and hence, in Northern Fennoscandia.

The next question is why is the 11-yr cycle did not manifest as obviously as the Gleissberg cycle. The answer can be taken from (Beer et al. 2000) where they constructed the solar irradiation spectrum at frequency time range from a $10^{3} \mathrm{yr}^{-1}$ to $10^{-1} \mathrm{yr}^{-1}$ and extrapolated the linear trend of the spectrum toward longer time scales. It was found that at the secular time scale, the amplitude of the irradiation variation is approximately three times larger than that induced by the 11-yr cycle. The reconstruction of the solar irradiation by Beer et al. (2000) for the period after $1700 \mathrm{AD}$ shows that the amplitude of 100 -yr variation can attain the value of about $4 \mathrm{~W} \times \mathrm{m}^{-2}$ (from $\sim 1353 \mathrm{~W} \times \mathrm{m}^{-2}$ in 1820 to $\sim 1357$ $\mathrm{W} \times \mathrm{m}^{-2}$ in 1900 [see Figure 7 in Beer et al. 2000]), while the amplitude of the 11-yr variation in irradiation, measured experimentally, is less than $2 \mathrm{~W} \times \mathrm{m}^{-2}$ (Frohlich and Lean 1997). Taking these results into account, the model suggested by Shindell et al. (1999) seems to be suitable for explanation of long-term solar forcing of climate in northern part of Northern Hemisphere.

\section{ACKNOWLEDGMENTS}

This work was completed through a framework of exchange between Russian and Finnish academies (project nr 16). G E Kocharov and M G Ogurtsov are thankful to the Russian Foundation for Fundamental Research for financial support (grant nr 99 02-18398). The authors are very grateful to Professor P Damon for his very detailed review of the paper and many useful recommendations for its improvement.

\section{REFERENCES}

Astafieva NM. 1996. Wavelet analyses: basic theory and some applications. UFN 166:1145-70. In Russian.

Beer J, Soos F, Lukachyk Ch. $1994 .{ }^{10} \mathrm{Be}$ as an indicator of solar variability and climate. In: Nesme-Ribes E, editor. The solar engine and its influence on terrestrial atmosphere and climate. NATO ASI_Series 25:221-33.
Beer J, Mende W, Stellmacher R. 2000. The role of the sun in climate forcing. Quaternary Science Reviews 19:403-15.

Damon PA, Sonett CP. 1991. Solar and terrestrial components of ${ }^{14} \mathrm{C}$ spectrum. In: Sonett CP, Giampapa MS, Mattheus MS, editors. The Sun in time. p 360-88. 
Damon PA, Peristykh AN. 1999. Solar cycle length and 20th century Northern Hemisphere warming. Geophys. Research Letters 26:2469-247.

Damon PA, Peristykh AN. 2000. Radiocarbon calibration and application to geophysics, solar physics, and astrophysics. Radiocarbon 42(1):137-50.

Dergachev VA, Veksler VS. 1990. Application of radiocarbon method for the investigation of past nature. Leningrad. 255 p. In Russian.

Friis-Christensen E, Lassen K. 1991. Length of the solar cycle an indicator of solar activity closely associated with climate. Science 254:698-700.

Frohlich C, Lean J. 1997. Total solar irradiance variations. In: Deubner FL, editor. IAU symposium 185: new eyes to see inside the sun and stars. Kyoto: Kluwer Academic Publications.

Gierens K, Ponater M. 1999. Comment on "Variation of cosmic ray flux and global cloud coverage - a missing link in solar-climate relationship" by H Svensmark and E Friis-Christiensen (1997). Journal of Atmospheric and Solar-Terrestrial Physics 61:795-7.

Gleissberg W. 1944. Terr. Mag. 49:243-4.

Grossman A, Morlet J. 1984. Decomposition of hardy functions into square integrable wavelets of constant shape. SIAM J. Math. Anal. 15:723.

Hoit DV, Schatten KH. 1997. The role of the sun in climate change. New York: Oxford University Press.

Jorgensen TS, Hansen AW. 2000. Comment on "Variation of cosmic ray flux and global cloud coverage - a missing link in solar-climate relationship" by $\mathrm{H}$ Svensmark and E Friis-Christiensen. Journal of Atmospheric and Solar-Terrestrial Physics 62:73-7.

Kocharov GE, Ostryakov VM, Chernov SS. 1981. Five reservoir model of carbon exchange cycle and some astrophysical phenomena. PTI Preprint-739. 26 p. In Russian.

Lindholm M, Eronen M. 2000. A reconstruction of midsummer temperatures from ring-widths of Scots pine since A.D. 50 in northern Fennoscandia. Geografisca Annaler, Series A. Forthcoming.

Mallatt SG. 1989. Multiresolution approximations and wavelet orthonormal bases of $\mathrm{L}^{2}(\mathrm{R})$. Transactions of the American Mathematical Society 315:69.

Mann ME, Bradley RS, Hughes MK. 1999. Northern Hemisphere temperatures during the past millennium inferences, uncertainties, and limitations. Geophys. Res. Letters 26(6):759.

Mitchell JM, Stockton CV, Meko DM. 1979. McCormac
BM, Seliga T, editors. Solar-terrestrial connections, weather and climate. Dordrecht: D. Reidel Publishing Co. p 125-43.

Monin AS. 1969. Weather forecast as the problem of physics. Moscow: Nauka. In Russian.

Nagovitsyn YuA. 2001. The Sun's activity for two last millennia A.D.: "solar monitoring" in the ancient and medieval China. Geomagnetism and Aeronomy. Forthcoming.

Pittock AB. 1978. A critical look at long-term sunweather relationship. Rev. Geoph. and Space Physics 16:400-20.

Persival DB. 1995. On estimation of the wavelet variance. Biometrika 82:619-31.

Pudovkin MI, Veretenenko SV. 1996. Variations of the cosmic rays as one of the possible link between the solar activity and the lower atmosphere. Advances in Space Research 17:161-4.

Shindell D, Rind D, Balachandran N et al. 1999. Solar cycle variability, ozone and climate. Science 284:305-8.

Schove DJ. 1983. Sunspot cycles. Stroudsburg: Hutchinson Ross.

Svensmark H, Friis-Christiensen E. 1997. Variation of cosmic ray flux and global cloud coverage - a missing link in solar-climate relationship. Journal of Atmospheric and Solar-Terrestrial Physics 59:1225-32.

Stuiver M, Pearson PJ. 1993. Extended ${ }^{14} \mathrm{C}$ data base and revised CALIB $3.0{ }^{14} \mathrm{C}$ age calibration program. $R a$ diocarbon 35(1):215-30.

Stuiver M, Braziunas TF, Becker B, Kromer B. 1991. Climatic, solar, oceanic and geomagnetic influences on late-glacial and holocene atmospheric ${ }^{14} \mathrm{C} /{ }^{12} \mathrm{C}$ changes. Quaternary Research 35:124.

Tinsley BA, Heelis RA. 1993. J. Geophys. Res. 98(D6): 10,375 .

Torrence C, Compo GP. 1998. A practical guide to wavelet analyses. Bulletin of American Meteorological Society 79:61-78.

Veretenenko SI, Pudovkin MI. 1993. Geomagnetism and Aeronomy 33(6):35.

Ulikh T. 1996. The varying length of the solar cycle as determined by different methods [MSc thesis]. University of Oulu, Finland.

Waldmeier M. 1961. The sunspot activity in the years 1610-1960. Zurikh: Schulthess and Company AG.

Wittmann AD, Xu Z. 1987. A catalogue of sunspot observations from $165 \mathrm{BC}$ to AD 1684. Astr. Astroph. Suppl. Ser. 70(1):83-94. 